University of Nebraska - Lincoln

DigitalCommons@University of Nebraska - Lincoln

\title{
$3-2013$
}

\section{High-Stress Shear-Induced Crystallization in Isotactic Polypropylene and Propylene/Ethylene Random Copolymers}

Zhe Ma

Eindhoven University of Technology, Z.Ma@tue.nl

Lucia Fernandez-Ballester

University of Nebraska-Lincoln, lucia.fernandez@unl.edu

Dario Cavallo

Eindhoven University of Technology, D.Cavallo1@tue.nl

Tim Gough

University of Bradford, UK, t.gough@bradford.ac.uk

Gerrit W. M. Peters

Eindhoven University of Technology, g.w.m.peters@tue.nl

Follow this and additional works at: https://digitalcommons.unl.edu/mechengfacpub

Part of the Engineering Science and Materials Commons, and the Polymer and Organic Materials Commons

Ma, Zhe; Fernandez-Ballester, Lucia; Cavallo, Dario; Gough, Tim; and Peters, Gerrit W. M., "High-Stress Shear-Induced Crystallization in Isotactic Polypropylene and Propylene/Ethylene Random Copolymers" (2013). Mechanical \& Materials Engineering Faculty Publications. 91.

https://digitalcommons.unl.edu/mechengfacpub/91

This Article is brought to you for free and open access by the Mechanical \& Materials Engineering, Department of at DigitalCommons@University of Nebraska - Lincoln. It has been accepted for inclusion in Mechanical \& Materials Engineering Faculty Publications by an authorized administrator of DigitalCommons@University of Nebraska Lincoln. 


\title{
High-Stress Shear-Induced Crystallization in Isotactic Polypropylene and Propylene/Ethylene Random Copolymers
}

\author{
Zhe Ma,,2 Lucia Fernandez-Ballester, ${ }^{2,3}$ Dario Cavallo,, ${ }^{1,2}$ Tim Gough, ${ }^{4}$ and Gerrit W. M. Peters ${ }^{1,2}$
}

1. Department of Mechanical Engineering, Eindhoven University of Technology, P.O. Box 513, 5600MB Eindhoven, The Netherlands 2. Dutch Polymer Institute (DPI), PO Box 902, 5600 AX Eindhoven, The Netherlands

3. DUBBLE at European Synchrotron Radiation Facility, 6 Rue Jules Horowitz, 38043 Grenoble, France; Present Address: Department of Mechanical and Materials Engineering and Nebraska Center for Materials and Nanoscience, University of Nebraska, Lincoln, Nebraska 68588, USA

4. School of Engineering, Design and Technology, University of Bradford, Bradford BD7 1DP, UK

Corresponding author - G.W.M. Peters, tel 31(0)402474840, email g.w.m.peters@tue.nl

\section{Abstract}

Crystallization of an isotactic polypropylene (iPP) homopolymer and two propylene/ethylene random copolymers (RACO), induced by high-stress shear, was studied using in situ synchrotron wide-angle X-ray diffraction (WAXD) at $137^{\circ} \mathrm{C}$. The "depth sectioning" method (Fernandez-Ballester, Journal of Rheology 53:5 (2009), pp. 1229-1254) was applied in order to isolate the contributions of different layers in the stress gradient direction and to relate specific structural evolution to the corresponding local stress. This approach gives quantitative results in terms of the specific length of fibrillar nuclei as a function of the applied stress. As expected, crystallization becomes faster with increasing stress - from the inner to the outer layer - for all three materials. Stress-induced crystallization in a RACO with $7.3 \mathrm{~mol} \%$
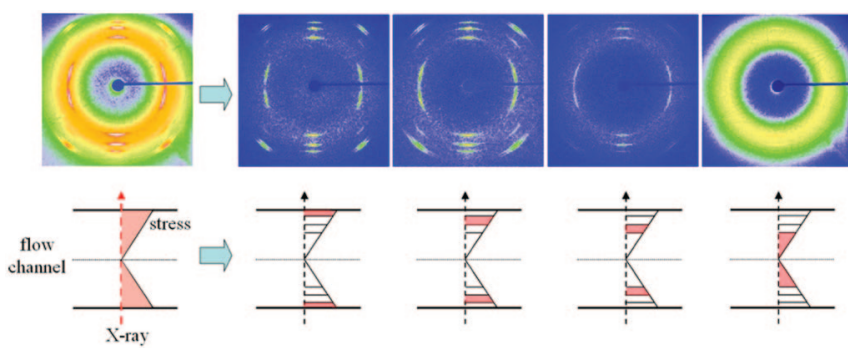
ethylene content was triggered at only $1{ }^{\circ} \mathrm{C}$ below its nominal melting temperature. The comparison of iPP and RACO's with 3.4 and 7.3 mol \% ethylene monomer reveals the effect of ethylene defects on high-stress shear induced crystallization at $137^{\circ} \mathrm{C}$. It is found that, for a given applied stress, the specific nuclei length formed by flow increases with ethylene content - which is attributed to a greater high molecular weight tail. However, the linear growth rate is significantly reduced by the presence of ethylene comonomers and it is found that this effect dominates the overall crystallization kinetics. Finally, a time lag is found between development of parent lamellae and the emergence of daughter lamellae, consistent with the concept of daughter lamellae nucleated by homoepitaxy on the lateral faces of existing parent lamellae.

\section{Introduction}

Semicrystalline polymers are usually processed from their molten state and subjected to intense shear and/or elongation flows. Such flow fields not only accelerate crystallization kinetics, which shortens the processing time, but can also change the morphology from isotropic spherulites to highly oriented shish-kebab structures ${ }^{1-3}$ and, as a consequence, determine the ultimate product properties. Therefore, understanding the interplay between strong flow fields and the resulting structures is of importance for designing processing procedures to tailor these end product properties.

Considerable work ${ }^{4-7}$ has been devoted to this topic in the past half century. Many researchers have focused on the relation between shear flow and polymer crystallization, because shear fields are easily created with rotational ${ }^{3}$ or sliding ${ }^{8,9}$ plate-plate devices, on rotational rheometers, ${ }^{10,11}$ and in pressure driven slit flows. ${ }^{12,13}$ These test geometries are typically combined with time-resolved characterization techniques like mechanical spectrometry, ${ }^{10,11,14}$ light scattering, ${ }^{15,16}$ birefringence, $^{13,17}$ X-ray scattering ${ }^{7,18,19}$ and Fourier transform in- frared (FTIR) spectroscopy, 9, 20, 21 etc. Significant progress has been made in understanding shear-induced crystallization, ${ }^{4-7}$ while some of the fundamental issues remain unsolved. In particular, knowledge of basic mechanism of crystallization under high shear rates and stress-close to realistic processing conditions - is still limited. The need for such information is becoming urgent in order to improve the latest simulation models, since the results of numerical predictions of, for example, injection molding, ${ }^{22}$ have to be validated and further refined from experimental evidence.

Imposing a strong shear flow at chosen high shear rates or stresses under well-defined conditions requires a specially designed flow device. Both the pressure-driven slit flow apparatus constructed by Janeschitz-Kriegl et al. ${ }^{12}$ and improved by Kornfield et al., ${ }^{13}$ and the piston-driven slit rheometer developed by Mackley et al. ${ }^{23}$ and modified by Peters and coworkers, ${ }^{24,25}$ can operate in the high stress region (of the order of $0.1 \mathrm{MPa}$ ) and are easily combined with time-resolved birefringence ${ }^{24}, 26$ and/or X-ray scattering $27-30$ measurement techniques. The drawback of these channel devices is 
the nonhomogeneous shear stress distribution over the sample thickness ${ }^{31}$ and, consequently, any observation in the shear gradient direction represents an average over the thickness of the sample. To solve this problem, Fernandez-Ballester et al. recently proposed and verified the "depth sectioning method", 30 which takes advantage of the linear variation of shear stress over the thickness, from a maximum and known shear stress at the wall to zero at the center of the rectangular channel. This method separates the contributions from specific layers by performing a series of experiments with varying wall stress but fixed shearing time.

In this work, the pressure-driven flow device ${ }^{13}$ and the "depth sectioning method" 30 are combined to quantitatively study polymer crystallization induced by high-stress shear. An isotactic polypropylene (iPP) and two propylene/ethylene random copolymers with various ethylene monomer contents are studied and compared to reveal the effect of molecular architecture. Recent studies found that adding ethylene monomer to the propylene chain can improve transparency, relative softness and low-temperature impact strength. ${ }^{32-34}$ Also, it has been found that the presence of ethylene monomer along the polypropylene chain disturbs the chain regularity and, consequently, decreases polymer crystallization ability; ${ }^{34-36}$ e.g., its presence decreases crystallinity and linear growth rate and, moreover, induces the formation of the orthorhombic $\gamma$-phase. ${ }^{36,37}$ However, the effects above have mostly been studied for quiescent crystallization, or under a rheometric flow ${ }^{35}$ unable to impose high shear stresses similar to those typically encountered in industrial processing conditions. Here, we focus on the effect of the presence of defects in the molecular architecture on shear-induced crystallization. Moreover, we show the importance of the high molecular weight tail on the effect of flow-induced crystallization.

\section{Experimental Section}

The materials used are an isotactic polypropylene (Borealis $\mathrm{HD} 234 \mathrm{CF}$ ) and two propylene/ethylene random copolymers (Borealis RD204CF and RD208CF), polymerized using Ziegler-Natta type catalysts. All three materials have very similar weight-average molecular mass $M_{\mathrm{w}} \approx 310 \mathrm{~kg} / \mathrm{mol}$ and a polydispersity of $M_{\mathrm{w}} / M_{\mathrm{n}} \approx 3.4$, but vary in their ethylene content between $0-7.3$ $\mathrm{mol} \%$. Their molecular and physical properties $\mathrm{s}^{35}$ are summarized in Table 1. In this study, the homopolymer is denoted as "iPP" while the copolymers are denoted as "RACO3" and "RACO7", according to their respective ethylene content in $\mathrm{mol} \%$.

Flow-induced crystallization experiments were carried out in a pressure-driven flow cell designed by Kumaraswamy et al. ${ }^{13}$ The flow cell, described previously, $13,26,30$ has a shear slit with a rectangular cross-section of $6.35 \mathrm{~mm}$ (width) $\times 0.5 \mathrm{~mm}$ (thickness) and a channel length of $63.5 \mathrm{~mm}$. It is equipped with two diamond windows mounted flush on the slit channel which allow the passage of an X-ray beam through the thickness of the sample for in situ measurements.

Table 1. Molecular and Physical Properties of iPP Homopolymer and Ethylene/Propylene Random Copolymers

\begin{tabular}{llllll}
\hline polymer & grade & $\begin{array}{l}\text { ethylene content } \\
(\mathrm{mol} \mathrm{\%})^{\mathrm{a}}\end{array}$ & $\begin{array}{l}X_{\mathrm{c}} \\
(\%)\end{array}$ & $\begin{array}{l}T_{\mathrm{m}} \\
\left({ }^{\circ} \mathrm{C}\right)^{\mathrm{b}}\end{array}$ & $\begin{array}{l}T_{\mathrm{c}} \\
\left({ }^{\mathrm{C}} \mathrm{C}\right)^{\mathrm{b}}\end{array}$ \\
\hline iPP & HD234CF & 0 & 48.3 & 159 & 110 \\
RACO3 & RD204CF & 3.4 & 42.3 & 147 & 105 \\
RACO7 & RD208CF & 7.3 & 34.4 & 138 & 98 \\
\hline
\end{tabular}

a. Measured by NMR.

$\mathrm{b}$. Measurements were performed under a heating and cooling rate of $10^{\circ} \mathrm{C} / \mathrm{min}$.
It is known that flow has a significant influence on crystallization, but which specific flow variable is the most dominant one is not clear yet. It is suggested that shear stress, ${ }^{26}$ shear strain ${ }^{19}$ and mechanical work ${ }^{38}$ can be the threshold criteria of formation of oriented structures. The correspondence between these variables involves viscosity which is a function of temperature. The three materials studied in this work have very close average melt rheological properties ${ }^{35}$ i.e., viscosity, at the same temperature. Therefore, to ensure that different polymers experience the same macroscopic flow in terms of all the above-mentioned variables, the same flow temperature $\left(137^{\circ} \mathrm{C}\right)$ was chosen for all materials. The experimental protocol is as follows: first, the material in the slit is heated to $215^{\circ} \mathrm{C}$ and kept at this temperature for $5 \mathrm{~min}$ to erase all thermal and mechanical history. Next, the relaxed melt is cooled to the desired crystallization temperature $T=137^{\circ} \mathrm{C}$. Once the sample is stabilized at $137^{\circ} \mathrm{C}$, a shear pulse is imposed on the molten polymer at a specific value of wall stress $(0.110,0.103,0.091$, and $0.079 \mathrm{MPa})$ for a fixed duration of $2 \mathrm{~s}$. The sample is held at $137^{\circ} \mathrm{C}$ after the shear pulse and the progress of crystallization under isothermal conditions is monitored by acquiring X-ray diffraction patterns. The depth sectioning method ${ }^{30}$ is then used on the diffraction patterns to isolate the structural information from various layers and relate this to the local stress (see below).

Time-resolved wide-angle X-ray diffraction (WAXD) characterization was carried out at the BM26B (DUBBLE) beamline ${ }^{39}$ at the European Synchrotron Radiation Facility (Grenoble, France) using a wavelength of $1.22 \AA$. Two-dimensional (2D) images were recorded with a Frelon detector with a resolution of $1024 \times 1024$ pixels of 97.6 $\mu \mathrm{m} \times 97.6 \mu \mathrm{m}$. The sample-to-detector distance was $157 \mathrm{~mm}$. The incoming beam intensity was measured with an ionization chamber to correct for changes in the primary beam intensity. The data acquisition time was $15 \mathrm{~s}$ per image. The shear pulse-lasting $2 \mathrm{~s}-$ was applied at the beginning of the acquisition of the first diffraction image. Therefore, this first image combines the information on $2 \mathrm{~s}$ of shear and of the subsequent $13 \mathrm{~s}$ of isothermal crystallization, and is noted as corresponding to a crystallization time of $13 \mathrm{~s}$.

The linear growth rates were measured by following quiescent melt-crystallization using a Leica DMLP polarized optical microscope equipped with a $20 \times$ objective lens. The microscope was coupled with a Linkam CSS450 stage to enable a careful control of the thermal history while acquiring optical micrographs with a dedicated digital video-camera. The samples were initially loaded in the cell as a pellet, melted and compressed into a film of approximately $20 \mu \mathrm{m}$ thick by moving the stage plates gently toward each other. The polymer films were annealed for $5 \mathrm{~min}$ at $210{ }^{\circ} \mathrm{C}$ and then cooled to the selected crystallization temperatures at a rate of $30{ }^{\circ} \mathrm{C} / \mathrm{min}$. Optical micrographs were taken during the isothermal crystallization, with adequate time-resolution. Spherulitic growth rate was determined by measuring the evolution of the spherulites diameter over time, by means of image analysis software ImageJ. The reported values of growth rate are the results averaged over three measurements and the reproducibility was within $\pm 3 \%$.

Depth Sectioning Method. The depth sectioning method 30 uses the linear relationship between layer depth from the wall and shear stress to separate the local structure in a specific layer, which is a prerequisite to reveal the relation between the shear history and the structural evolution. For pressure driven flow, the shear stress varies linearly along the channel thickness direction from zero on the center line to maxima at the walls (see Figure 1). Because $X$-rays propagate through the sample along the thickness direction, i.e., the stress gradient direction, the acquired X-ray patterns correspond to the total diffraction from all layers. In order to apply the depth sectioning method and separate the diffraction signal corresponding to a specific sample layer, a set of experiments is performed at different wall shear stresses while keeping all other parameters fixed (e.g., temperature, shear duration, crystallization time).

Consider an experiment in which a wall shear stress of $\sigma_{\max }$ is applied and for which the corresponding scattering X-ray signal is $I_{\text {max }}^{\text {mot }}\left(\right.$ Figure ${ }^{1}$ ). For this experiment, the local shear stress $\sigma_{d}$ at a specific depth $d$ with respect to the nearest wall is given by: 


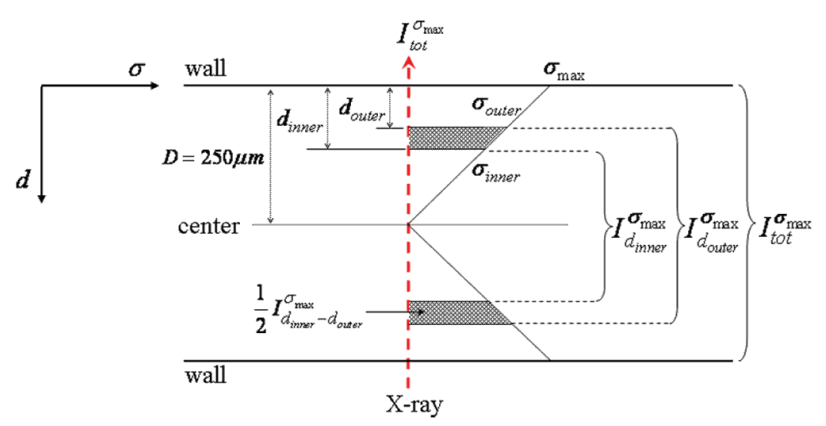

Figure 1. Schematic of the linear relationship between layer depth with respect to the wall $d$, and its local stress, $\sigma_{d}$.

$$
\frac{\sigma_{d}}{\sigma_{\max }}=\frac{D-d}{D}
$$

where $D$ corresponds to half the channel width $(250 \mu \mathrm{m}$ in the current experiments). According to the depth sectioning method, ${ }^{30}$ the contribution of the scattering signal arising from the interior portion between two boundaries at a depth $d$ from each wall, $I_{d}^{\sigma_{\max }}$, can be determined by performing another experiment in which a wall shear stress of $\sigma_{d}$ is imposed, and by subsequently rescaling the obtained scattering signal $I_{\text {tot }}^{\sigma_{d}}$ by the stress ratio, i.e., $I_{d}^{\sigma_{\max }}=I_{\text {tot }}^{\sigma_{d}} \times\left(\sigma_{d}\right) /\left(\sigma_{\max }\right)$.

As shown in Figure 1 , we can consider a specific layer that has two boundaries, $d_{\text {outer }}$ and $d_{\text {inner }}$, which correspond to specific shear stresses $\sigma_{\text {outer }}$ and $\sigma_{\text {inner }}$ respectively. According to the depth sectioning method, ${ }^{30}$ the contribution to scattering of the layer located between $d_{\text {outer }}$ and $d_{\text {inner }} I_{\text {dinner }}^{\sigma_{\max }}$ - douter to the total intensity measured for an experiment where a wall stress $\sigma_{\max }$ is imposed $\left(I_{\text {tot }}^{\sigma_{\max }}\right)$, can be determined by:

$$
I_{d_{\text {inner }}-d_{\text {outer }}}^{\sigma_{\max }}=I_{d_{\text {outer }}}^{\sigma_{\max }}-I_{d_{\text {inner }}}^{\sigma_{\max }}=I_{\text {tot }}^{\sigma_{\text {outer }}} \times \frac{\sigma_{\text {outer }}}{\sigma_{\max }}-I_{\text {tot }}^{\sigma_{\text {inner }}} \times \frac{\sigma_{\text {inner }}}{\sigma_{\max }}
$$

where $I_{\text {tot }}^{\sigma_{\text {outer }}}$ and $I_{\text {tot }}^{\sigma_{\text {inner }}}$ correspond to the total intensity signals obtained from two separated experiments using prescribed wall stresses of $\sigma_{\text {outer }}$ and $\sigma_{\text {inner, }}$ respectively.

A series of experiments with wall stresses of 0.110, 0.103, 0.091, and $0.079 \mathrm{MPa}$ were carried out to isolate four layers at depths of 0-16 (L1), 16-43 (L2), 43-70 (L3), and 70-250 $\mu \mathrm{m}$ (L4) from the wall. An example of depth-sectioned patterns for iPP after $88 \mathrm{~s}$ of isothermal crystallization is shown in Figure ${ }^{2}$. Because of the relatively high stress, crystallinity develops fast in the outer layers L1 and L2, where L1 has a higher orientation. The core part
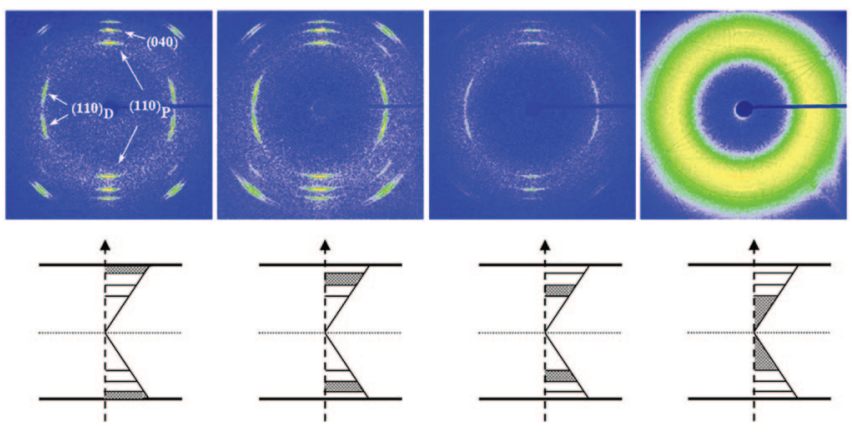

L1: $0-16 \mu \mathrm{m}$

$0.110-0.103 \mathrm{MPa}$
L3: $43-70 \mu \mathrm{m}$

L4: $70-250 \mu \mathrm{m}$

$0.103-0.091 \mathrm{MPa}$

$0.091-0.079 \mathrm{MPa}$

$0.079-0 \mathrm{MPa}$
Figure 2. 2D depth-sectioned diffraction patterns (top row) corresponding to the crystallization of specific layers (bottom row) in iPP at $t=88 \mathrm{~s}$ after a wall shear pulse of $0.11 \mathrm{MPa}$ and $2 \mathrm{~s}$. The layer depths and corresponding boundary stresses are indicated. Flow direction is horizontal.

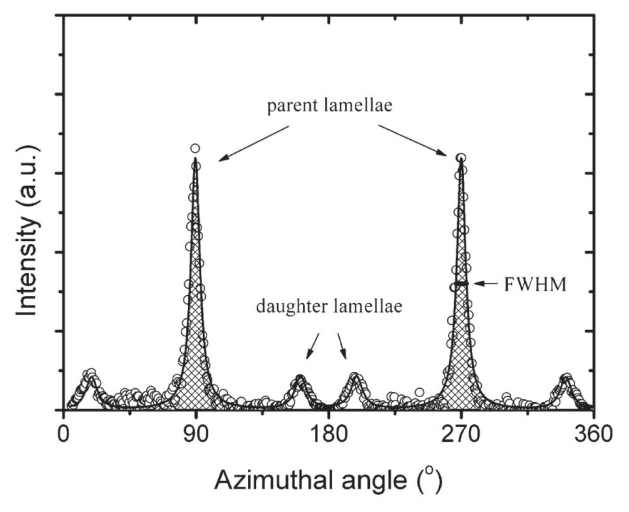

Figure 3. Azimuthal scan of the oriented (110) diffraction of iPP at $t$ $=88 \mathrm{~s}$ for the L1 layer after thickness normalization and geometrical correction. Open symbols are experimental data. The solid line corresponds to the sum of all separate Lorentzian fittings of parent and daughter lamellae. The filled region indicates the integral area of peak fittings of parent lamellae $(110)_{\mathrm{P}}$.

experiences the lowest stress, so in the L4 layer the polymer is still mainly in the amorphous state after $88 \mathrm{~s}$.

In order to enable the comparison of crystallization between the different layers, the depth sectioned intensities are further normalized by the thickness of each layer $\Delta d=d_{\text {outer }}-d_{\text {inner }}$. The WAXD diffractions in Figure 2 indicate formation of the monoclinic a-phase of isotactic polypropylene. The iPP a-phase can form two types of lamellar crystals with different orientations; parent lamellae and their epitaxial daughter lamellae. In the oriented case, parent and daughter lamellae can be distinguished according to their various orientations, i.e. the distinct azimuthal locations of (110) reflections between parent lamellae $(110)_{\mathrm{P}}$ and daughter lamellae $(110)_{\mathrm{D}}$ as shown in Figure 2. The information on parent lamellae can be extracted by fitting the azimuthal scan of the (110) diffraction arising from oriented crystals ${ }^{30}$ after subtraction of the isotropic part calculated from the oriented (040) diffraction and after application of the geometrical correction. ${ }^{40}$ As an example, the results for layer L1 are given in Figure 3. The (110) diffraction area $\left(\right.$ Area $\left._{\mathrm{P}, 110}\right)$ and full width at half-maximum (fwhm) from the parent lamellae can be determined and represent relative measures of the amount and of the orientation of parent crystallites, respectively.

\section{Results and Discussion}

The depth-sectioned X-ray patterns are first used to examine the influence of shear stress on the crystallization kinetics and orientation of each of the three materials; the homopolymer (iPP) and two random copolymers (RACO3 and RACO7). Next, the crystallization kinetics of these three different polymers is compared at specific levels of shear stress to reveal the effect of the macromolecular architecture-i.e., copolymer content - on crystallization.

iPP Homopolymer. Prior to flow, the diffraction pattern of the iPP presents only a broad isotropic ring (data not shown), irrespective of the layer, i.e., with application of depth sectioning. This is consistent with the undeformed amorphous melt with no crystallinity and no orientation. Selected 2D WAXD depth sectioned patterns during shear and following isothermal crystallization of the iPP for various layers are shown in Figure 4.

The results clearly show that stress has a remarkable influence on triggering crystallization. The different layers, from L1 to L3, exhibit a variety of crystallization behaviors because of the decreasing local stress. Figure 4-L1 presents the structural evolution in the outermost layer L1, subject to the highest 


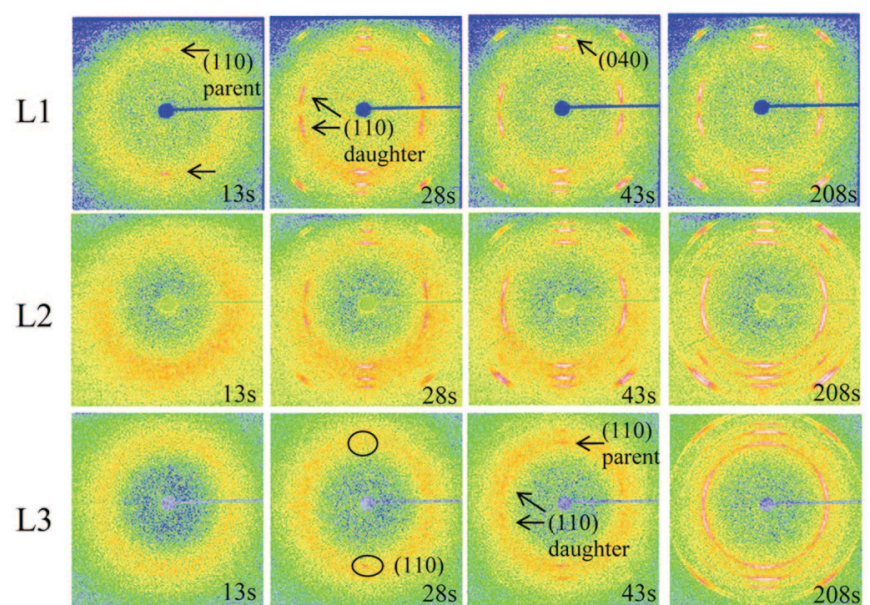

Figure 4. WAXD depth-sectioned patterns of isothermal iPP crystallization at $137^{\circ} \mathrm{C}$ for a wall shear stress of $0.110 \mathrm{MPa}$ for $2 \mathrm{~s}$. The positions and corresponding stresses of layer L1 (top row), L2 (middle row) and L3 (bottom row) are given by Figure 2. The flow direction is horizontal. The color scale in this figure has been adjusted to enable the observation of the weakest diffraction peaks.

stress range $0.11-0.103 \mathrm{MPa}$. A pair of clear arc-like diffractions emerge quickly after flow at a scattering vector of $q=$ $10.0 \mathrm{~nm}^{-1}$ in the vertical direction perpendicular to shear (Figure 4-L1, $13 \mathrm{~s}$ ). These WAXD diffraction arcs correspond to the (110) diffraction plane ${ }^{41}$ of the monoclinic a-phase in iPP, indicating that the oriented a-phase forms quickly after shear and that the $c$-axis aligns along the flow direction in the so-called parent lamellae. In contrast, crystallization under lower shear stresses in the L2 and L3 layer is more sluggish and requires a longer time, around $28 \mathrm{~s}$, to form detectable parent crystals (Figure 4, parts L2 and L3). Likewise, in the two outermost layers L1 and L2, daughter lamellae-also described as lamellar branches at an angle of $80^{\circ}$ to a specific parent lamellar surface $^{42}$ - are already observed at $28 \mathrm{~s}$ as two pairs of (110) diffraction arcs located close to the meridian (Figure 4, parts L1, $28 \mathrm{~s}$, and L2, $28 \mathrm{~s}$ ). In contrast, for the L3 layer, daughter lamellae only appear at later times (43 s).

It should be noted that only the a-phase appears in the 2D diffraction patterns. Although some studies have observed the emergence of considerable $\beta$-phase ${ }^{43,44}$ and $\gamma$-phase ${ }^{45}$ crystals in shear-induced iPP crystallization, the appearance of only a-phase in our results is consistent with previous studies that found only or predominantly a-phase as a result of shear-induced crystallization. ${ }^{17,} 28,30$

The results in Figure 4 indicate that the imposition of a shear pulse generates shear-induced nuclei which can significantly speed up crystallization kinetics and orient the crystal morphology. ${ }^{12,19}, 46$ At $137^{\circ} \mathrm{C}$, quiescent crystallization of iPP is too slow to generate any detectable structure within 400 s (data not shown), and only isotropic crystallites would ultimately form. In contrast, the diffraction patterns at $208 \mathrm{~s}$ for the L1 layer are quite narrow in the azimuthal direction (see Figure 4-L1, 208 s) implying that crystal morphology in the layer that was subjected to the highest stress range is highly oriented. For the inner layers subjected to lower levels of shear stress, however, the orientation of structures is qualitatively lower at 208 s. Therefore, depth-sectioned WAXD images qualitatively show that stress has a significant influence on the start and evolution of crystallization.

Next, a quantitative evolution of the amount of parent-lamellar crystals and the degree of orientation is extracted from the area $\left(\right.$ Area $\left._{P, 110}\right)$ and fwhm of the azimuthal (110) peak corresponding to the parent lamellae (see Figure 5, parts a and $b$ ). Irrespective of the layer considered, parent lamellae grow rapidly in the early stages and then reach a shoulder, after which they either halt their growth or they continue to grow at a much slower rate. Knowing that the growth is stopped by the impingement of the growth fronts of the parent lamellae, a shorter time to reach this shoulder must relate to less space between neighboring nuclei. Therefore, more nuclei are generated in the outer layer by the higher stress. ${ }^{26,30,47}$ Interestingly, the crystallization in the L1 layer not only shows the fastest kinetics at the early stages, but also possesses the highest amount of parent crystallites (Area ${ }_{\mathrm{P}, 110}$ value) when it reaches the shoulder. The larger value may result from the combined effect of flow-raised crystallinity and parent/daughter lamellae ratio $^{28,30}$ when crystallization is completed, as found by Fernandez-Ballester et al. ${ }^{30}$ and $\mathrm{Ku}$ maraswamy et $a .^{28}$ that the relative ratio between parent and daughter lamellae is higher in the outer layer than in the inner layers of lower stress.

Shear-induced nuclei are known to template the oriented growth of parent lamellae in the early stage. ${ }^{28,30}$ The orientation of parent lamellae was illustrated by the fwhm of the parent (110) diffraction, see Figure 5b. Lower fwhm values refer to a higher average lamellar orientation. The fwhm in the L1 layer is the lowest, around $6^{\circ}$, but the larger ones in the L2 and L3 layers are quite similar $\left(\approx 9^{\circ}\right)$ in the first
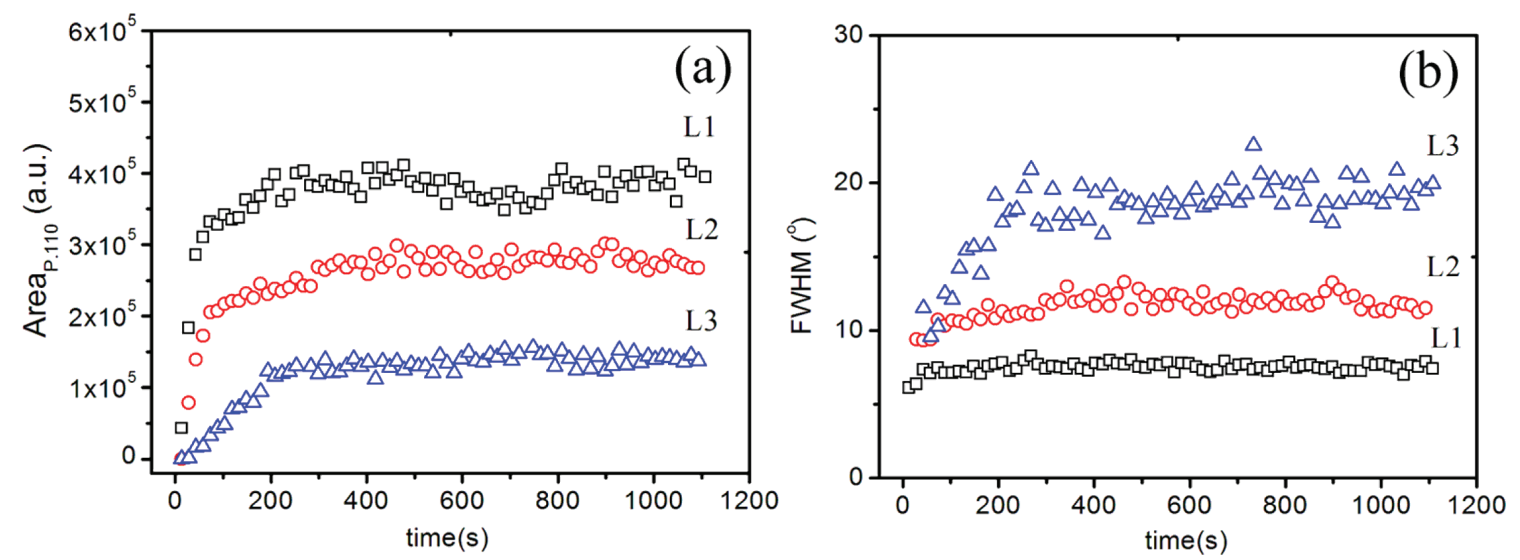

Figure 5. Evolution of area (a) and fwhm (b) of parent (110) diffraction in iPP isothermal crystallization for the different layers. Layer positions and corresponding stresses are given by Figure 2 . 
short period of tens of seconds. These low fwhm values in the initial stage of crystallization suggest that nuclei generated at various stress levels have a high orientation. This result is consistent with the observation of Fernandez-Ballester et al., ${ }^{30}$ where crystallites induced by the three strongest conditions show very high and similar degrees of orientation at the beginning (see Figure 9b in Reference 30: Fernandez-Ballester, Thurman, \& Kornfield, J. Rheol. 53:5 [2009], pp. 1229-1254).

As crystallization proceeds, the change in the orientation becomes more pronounced for the inner layers with lower shear stress. For the highest stress range of $0.110-0.103 \mathrm{MPa}$ the fwhm remains nearly constant during the observation period, whereas those for 0.103-0.091 and 0.091-0.079 MPa vary from $9^{\circ}$ to $12^{\circ}$ and from $9^{\circ}$ to $19^{\circ}$, respectively. The change in the orientation indicates that lamellar growth does not strictly follow that of the nuclei or initially grown lamellae because of the occurrence of lamellar curving and twisting during lateral growth. ${ }^{40}$ This orientation variation depends on the space between neighboring nuclei. When nuclei density is lower, there exists more space between nuclei for lateral growth during which the possibility to curve increases leading to the reduction of orientation. ${ }^{30,48}$ Therefore, orientation evolution shows that the L3 layer has the least nuclei, which is consistent with the results of area evolution.

Propylene/Ethylene Random Copolymers (RACO3 and RAC07). Only three out of the four wall shear stresses for the homopolymer were imposed on the random copolymers $(0.110,0.103$, and $0.091 \mathrm{MPa})$. For the random copolymers, the crystallization at a wall stress of $0.091 \mathrm{MPa}$ was quite sluggish, so the stress was not lowered further to $0.079 \mathrm{MPa}$. Therefore, the innermost layer of random copolymers (named L3 +4 , see Figure 6) should be compared to the sum of the two individual L3 and L4 layers in iPP. Note that at the same experimental temperature $T_{\text {exp }^{\prime}}$ iPP and random copolymers have different undercooling, $\Delta T=T_{m}{ }^{o}-T_{\exp ^{\prime}}$ where $T_{m}{ }^{0}$ is the equilibrium melting temperature, because the addition of ethylene monomer decreases the equilibrium melting temperature $T_{m}{ }^{0}{ }^{35} \mathrm{Ac}-$ cordingly, the lamellar linear growth rate under quiescent conditions decreases with the increase of ethylene content (see the Discussion section).

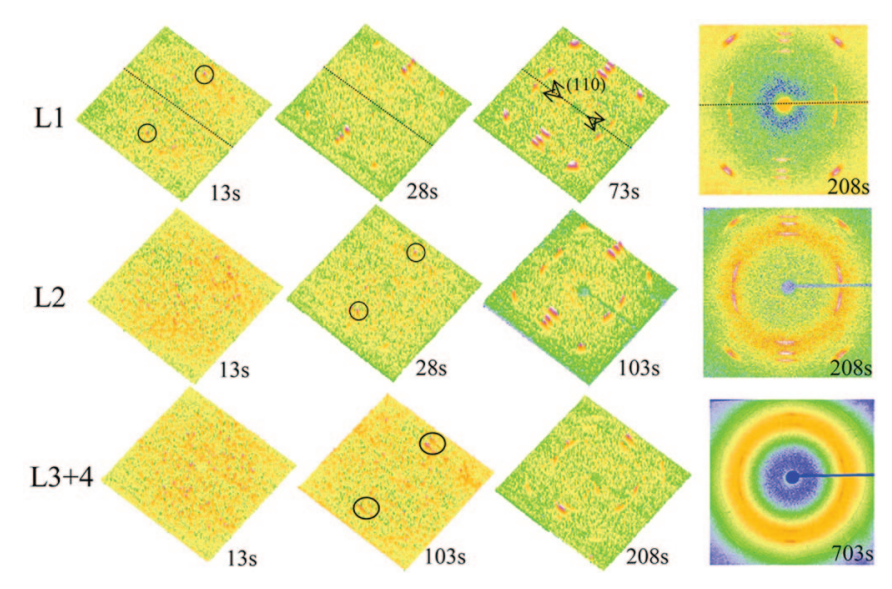

Figure 6. WAXD depth-sectioned patterns of isothermal RACO3 crystallization at $137^{\circ} \mathrm{C}$ for a wall shear stress of $0.11 \mathrm{MPa}$ for $2 \mathrm{~s}$. The positions and stresses of layer L1 (top row), L2 (middle row), and L3 + 4 (bottom row) are given by Figure 2. The flow direction is horizontal. Images are tilted to make the weak diffraction patterns more clear.
Figure 6 shows a representative series of depth-sectioned WAXD patterns for RACO3. The influence of stress is also found to be significant for RACO3. In the outermost L1 layer, some oriented crystallites can already be observed within the first $13 \mathrm{~s}$ after imposing the shear pulse, while in the inner L2 and L3 + 4 layers, crystallites can only be detected after 28 and $103 \mathrm{~s}$, respectively. The faster kinetics in the outer layer indicates that, as for iPP, increasing applied shear stress induces more nuclei also for RACO3. Comparing with iPP, the crystallization of RACO3 in the L1 and L2 layer starts at the approximately same time (13 and $28 \mathrm{~s}$, respectively), but in the L3 +4 layer it is much slower than for iPP in the L3 layer (see L3, 28 s, in Figure 4 and L3 + 4, 103 s, in Figure 6).

Interestingly, all layers of RACO3 show a time lag between the development of parent and daughter lamellae. For instance, in the L1 layer, RACO3 parent lamellae development is pronounced from 13 to $28 \mathrm{~s}$, while no daughter lamellae are observed at all at $28 \mathrm{~s}$. Similarly, for low stresses (Figure 6-L2, $28 \mathrm{~s}$, and Figure 6-L3 + 4, $103 \mathrm{~s}$ ) the first crystals that develop after flow belong to the parent lamellae only. Note that the third column in Figure 6 just shows typical WAXD diffractions during crystallization, rather than the first observations of daughter lamellae. The time lags (between appearances of parent and daughter lamellae) of RACO3 are around $30 \mathrm{~s}$ (which corresponds to two WAXD frames with acquisition period of $15 \mathrm{~s}$ per frame) for layers L1 and L2, while that of layer L3 +4 can not be precisely quantified due to the weak signal of daughter lamellae (data not shown). Considering the relatively long data acquisition time per frame, the influence of stress on the parent-daughter time lag of different layers is not discussed. This growth lag between different lamellae is not specific for RACO3; it is also observed for iPP in the L3 layer (Figure 4-L3, 28 s) and for RACO7 in the L1 layer (data not shown). In fact, this time lag is consistent with the mechanism of initiation of parent and daughter lamellae. Parent lamellae are templated from shear-induced nuclei, while daughter lamellae are nucleated by the homoepitaxy on the lateral (010) faces of existing parent lamellae with monoclinic a-modification. ${ }^{42}$ In other words, daughter lamellae need parent lamellae to initiate the second-generation growth.

Quantitative evolutions of parent lamellae are shown in Figure 7a, which indicates that the growth of oriented parent crystallites in layers L1 and L2 occurs rapidly after flow, fills the space and slows down due to the impingement of the growth fronts. The kinetics will be used to quantify the nuclei density in the next section. The rescaled inset in Figure 7a shows that crystallization in layer L3 + 4 does mainly occur by the flow-induced nuclei, consistent with the $2 \mathrm{D}$ images in Figure 6. However, flow-induced nuclei will not be quantified for layer L3 +4 because the evolution of oriented crystallites does not reach a plateau within the experimental time. The layer L3 +4 encompasses a wide interval of stress $(0-0.091 \mathrm{MPa})$. At low levels of stress, the density of flow-induced oriented nuclei is expected to be lower than at higher levels of stress; hence conditions of impingement between lamellar growing off nearby nuclei would be expected to take much longer time.

RACO3 orientation evolution is shown in Figure 7b. Layers L1 and L2 have a quite similar orientation at the start but develop differently with time. This is qualitatively consistent with the difference in nuclei density in the different layers; again, the larger space between neighboring nuclei allows for curving and twisting resulting in a lower orientation (a larger fwhm value). 

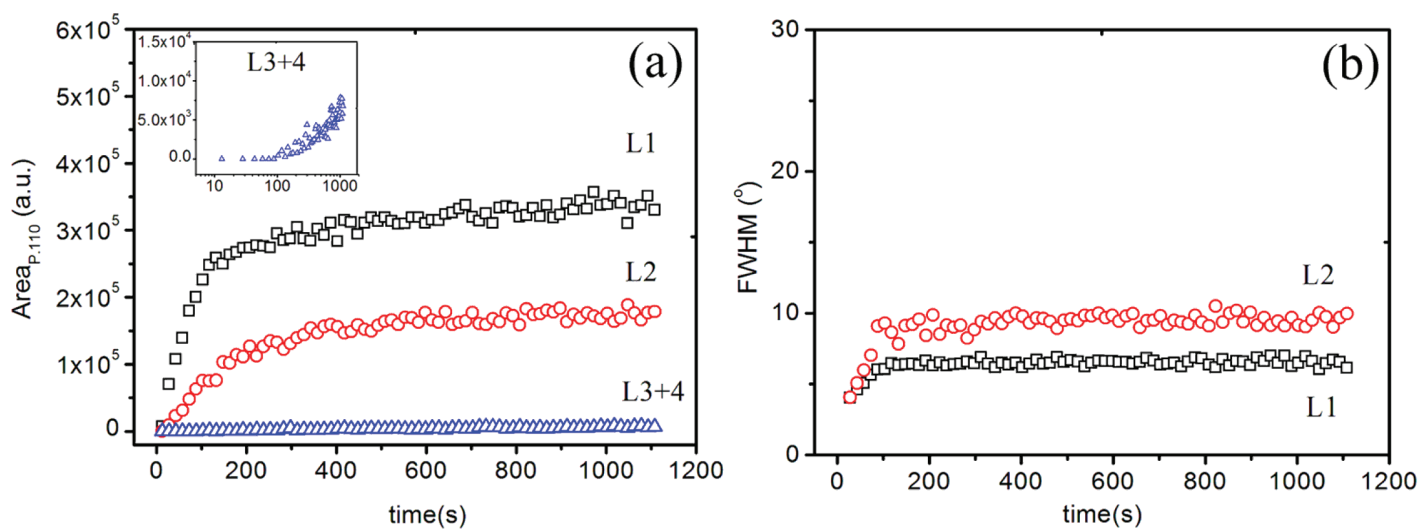

Figure 7. Evolution of area (a) and fwhm (b) of parent (110) diffraction in RACO3 during shear and isothermal crystallization. Layer positions and stresses are given by Figure 2.
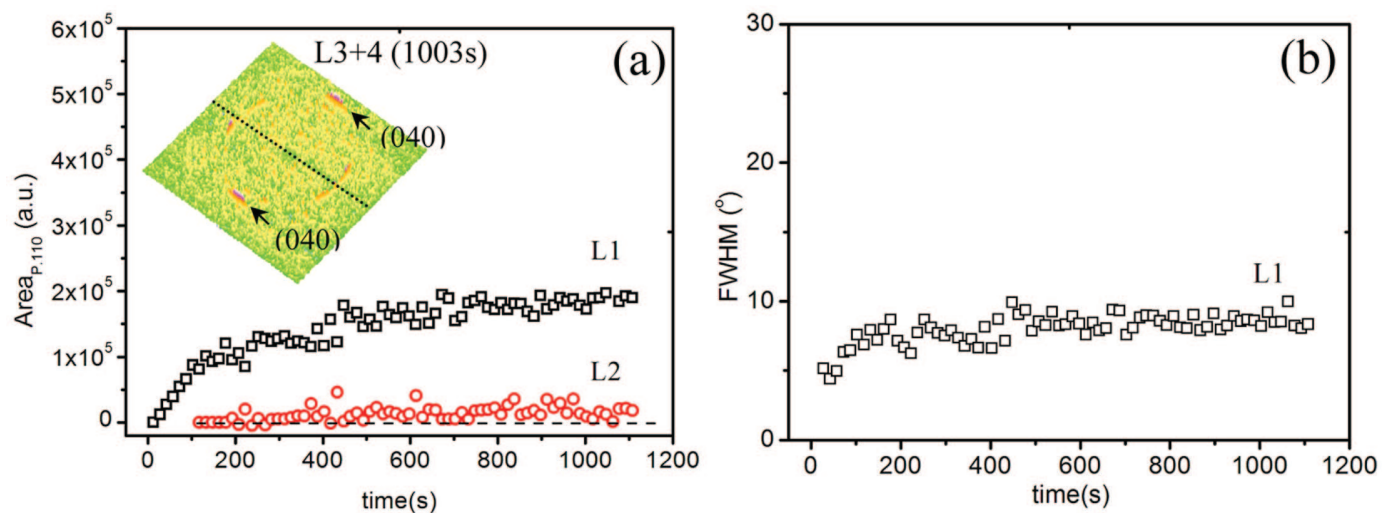

Figure 8. Evolution of area (a) and fwhm (b) of parent (110) diffraction in RACO7 during shear and isothermal crystallization. Inset is the WAXD image for RACO7 after crystallization for 1003 s. Layer positions and stresses are given by Figure 2.

For an ethylene content of $7.3 \mathrm{~mol} \%$ in RACO7, the nominal melting temperature decreases to $138^{\circ} \mathrm{C}$. Quiescent crystallization will not be detected at the experimental temperature of $137{ }^{\circ} \mathrm{C}$, since this is just $1{ }^{\circ} \mathrm{C}$ lower than its nominal melting temperature and, as a consequence, the linear growth rate is very small. However, crystallization of RACO7 in L1 layer proceeds immediately after flow and for the L2 layer, some crystallites become observable at around $200 \mathrm{~s}$ (Figure $8 a)$, providing a clear example of the effect of shear stress on crystallization even in the vicinity of the nominal melting temperature. The linear growth rate is the same as under quiescent conditions, so the accelerated rate of oriented crystallization results from the abundant oriented nuclei generated by the high shear stresses applied.

Comparing with iPP and RACO3, the time at which crystallization can first be detected for RACO7 is similar in L1 but much slower in L2 and L3. During crystallization, fwhm varies from $\sim 5^{\circ}$ to $\sim 8^{\circ}$, see Figure $8 \mathrm{~b}$. Interestingly, the slow RACO7 crystallization in the L1 layer is comparable to that of iPP in the L3 layer, but the orientation in RACO7 is much higher.

On the basis of above results, a qualitative conclusion can be drawn that for each of the three materials: the number of nuclei formed increases with applied shear stress, i.e., from the inner to the outer layers. For a given material, the comparison is simple because of the quiescent growth rate of lamellae is fixed. However, polymers with the various ethylene contents have different quiescent growth rates which affect the crystallization kinetics. Therefore, to quantitatively study the effects of stress and ethylene content on polymer crystallization, the kinetic model ${ }^{49-52}$ described below is used to estimate the amount of oriented nuclei formed by shear in the different materials below.

Quantification of Nuclei. In the Kolmogorov-Avrami-Evans model, ${ }^{49-52}$ the progress of space filling in time, $\Phi(t)$, can be described by the expression:

$$
\Phi(t)=1-\exp \left(-k G^{m} t^{n}\right)
$$

where $k$ is the factor involving the nuclei density, $G$ the linear growth rate, $m$ the exponent indicating the growth dimension (1-, 2-, or 3-dimensional) and $n$ the nucleation mechanism (sporadic, $n=m+1$ or predetermined, $n=m$ ). For shear-induced crystallization, the number of nuclei is fixed prior to growth and does not increase with space filling, so the exponent number $n$ equals the growth dimension $m$. In the present work, oriented nuclei are dominant and space is mainly filled by the lamellar growth that develops perpendicular to these nuclei. Structural "perfection" (e.g., lamellar perfection, branching and thickening) behind the growth front is not taken into account for space filling. Therefore, we assume that the space filling $\Phi(t)$ is directly proportional to the development of the parent lamellae diffraction $A(t)$. With this assumption, space filling can be quantified using

$$
\Phi(t)=\left(A(t)-A_{0}\right) /\left(A_{\infty}-A_{0}\right)
$$

where $A_{0}$ is the (110) diffraction area from parent lamellae at $t$ $=0$ that is caused by flow and $A_{\infty}$ is the (110) diffraction area at the shoulder when space filling is completed. ${ }^{53}$ Since the first data point for the L1 layer is obtained after flow and non- 


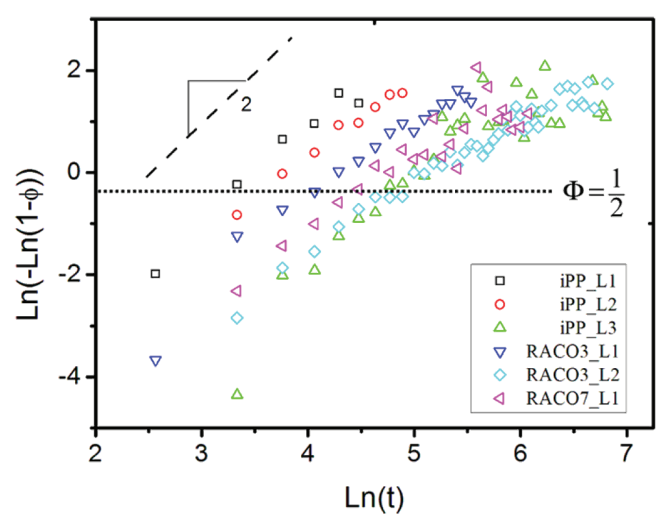

Figure 9. Avrami plots of space filling evolution for different materials in various layers.

zero, it contains information concerning both the $2 \mathrm{~s}$ of shear and the $13 \mathrm{~s}$ of isothermal crystallization, $A_{0}$ cannot be determined directly for all L1 layer cases. On the other hand, for most of the results in the L1 layer, after crystallizing for $13 \mathrm{~s}$, $A(13 \mathrm{~s})$ is still very low with respective to the shoulder value, so the contribution of $A_{0}$ to space filling is negligible and will be assumed to be 0 in the calculation of space filling. Therefore, space filling can be assessed by $\Phi(t)=A(t) / A_{\infty}$.

Assuming that the linear growth rate $G$ is constant in time, the crystallization kinetics can be examined by plotting the rewritten form of Equation 3 (see Figure 9):

$$
\ln \{-\ln [1-\Phi(t)]\}=n \ln (t)+\ln \left(k G^{m}\right)
$$

The fitted exponent are all in the range 1.6-2 $(n=2$ is for ideal $2 \mathrm{D}$ growth with predetermined nuclei) while all initial slopes are nearly 2 . Therefore, the theoretical integer exponent $n=2$ will be used for the assessment of nucleation density. The description of 2-dimensional growth reads thus: ${ }^{54}$

$$
\Phi(t)=1-\exp \left(-\pi l N G^{2} t^{2}\right)
$$

where $l$ is the long period of stacked lamellae, and $N$ is the number density of nucleation sites, i.e., the number of lamellae per volume. The total length of nuclei per volume, $L$, can be easily derived from the time for filling half space, $t_{1 / 2}$ :

$$
l \times N=L=\frac{\ln 2}{\pi\left(G \times t_{1 / 2}\right)^{2}}
$$

Note that for the random copolymers the addition of ethylene leads to defects in the regular polypropylene chain and, consequently, decreases the crystallization ability and the linear growth rate $G$.

The quiescent growth rates for the three materials at different temperatures are plotted in Figure 10. Because the measured temperature range is limited, a linear function ${ }^{55}(\log (\mathrm{G})$ vs. T) is used to estimate growth rates at $137^{\circ} \mathrm{C}$ (see Supporting Information), obtaining 24.5, 4.4, and $2.1 \mathrm{~nm} / \mathrm{s}$ for iPP, RACO3 and RACO7, respectively. Using these growth rate values, we estimated lengths of the oriented nuclei per volume given in Table 2.

For each material, the estimated oriented nuclei length per volume increases with increasing stress, i.e. from the inner to the outer layers, consistent with the trend of faster overall crystallization in the outer layers. In iPP, the oriented nuclei length per volume generated by the highest levels of stress is of the order of $10^{11} \mathrm{~m} / \mathrm{m}^{3}\left(0.1 \mu \mathrm{m} / \mu \mathrm{m}^{3}\right.$ in Table 2). Since the normal long period of iPP is typically tens of nanometers, ${ }^{56}$ the number of lamellae growing directly on the oriented

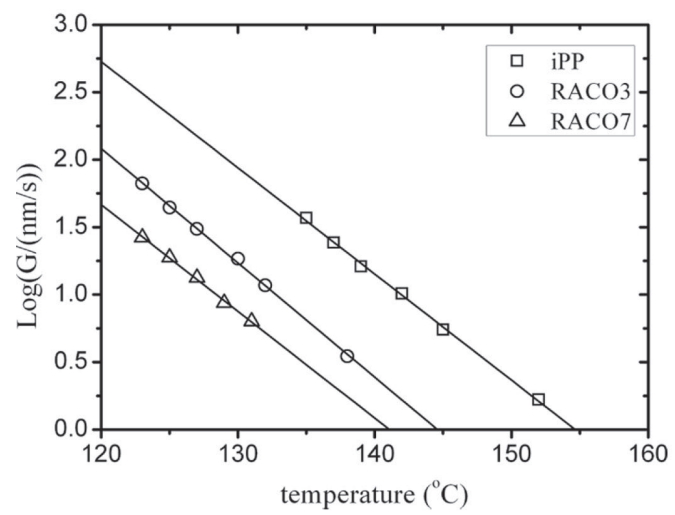

Figure 10. Growth rates measured with polarized optical microscopy at different temperatures under quiescent conditions. Open points are experimental data and solid lines represent linear fittings.

Table 2. Total Length per Volume (L) of Oriented Nuclei Calculated for iPP, RACO3, and RACO7 for Different Layers ${ }^{\mathrm{a}}$

\begin{tabular}{llll}
\hline $\begin{array}{l}\text { Total length } \\
\text { per volume of } \\
\text { oriented nuclei }\end{array}$ & iPP & RACO3 & RACO7 \\
\hline L1 & $0.54 \mu \mathrm{m} / \mu \mathrm{m}^{3}$ & $3.4 \mu \mathrm{m} / \mu^{3}$ & $6.9 \mu \mathrm{m} / \mu^{3}$ \\
& $(26 \mathrm{~s})$ & $(58 \mathrm{~s})$ & $(85 \mathrm{~s})$ \\
L2 & $0.28 \mu \mathrm{m} / \mu \mathrm{m}^{3}$ & $0.89 \mu \mathrm{m} / \mu \mathrm{m}^{3}$ & - \\
& $(36 \mathrm{~s})$ & $(113 \mathrm{~s})$ & \\
L3 & $0.026 \mu \mathrm{m} / \mu \mathrm{m}^{3}$ & - & - \\
& $(120 \mathrm{~s})$ & & \\
\hline
\end{tabular}

a. The estimated times for filling half space $\left(t_{1 / 2}\right)$ are presented in parentheses.

nuclei should be of the order of $10^{19} 1 / \mathrm{m}^{3}$, i.e., there are $\sim 10^{19}$ nucleating sites $/ \mathrm{m}^{3}$. Such large density of nucleating sites is 3 orders of magnitude greater than the highest nucleating density observed in previous studies under flow conditions that induce only point-like nuclei, $\sim 10^{16}$ nucleating sites $/ \mathrm{m}^{3}$ (estimated by considering that each shear-induced point-nuclei grows one spherulite). ${ }^{47}$ Alternatively, nuclei densities of up to $10^{19} 1 / \mathrm{m}^{3}$ can be achieved under quiescent conditions if an efficient nucleating agent is used. ${ }^{57}$

For random copolymers, the high stresses of 0.110 and $0.103 \mathrm{MPa}$ are able to trigger significant crystallization for a small degree of undercooling, particularly for RACO7, since the experimental crystallization temperature is just $1{ }^{\circ} \mathrm{C}$ below its nominal melting temperature. This effect is comparable with that observed for iPP when stresses between 0.08 and $0.19 \mathrm{MPa}$ are imposed at $165^{\circ} \mathrm{C},{ }^{29} 2^{\circ} \mathrm{C}$ above its nominal melting temperature. Therefore, polymer crystallization can be initiated even in the vicinity of the nominal melting temperature when the stress is high enough, i.e. in the order of $0.1 \mathrm{MPa}$ for RACO7.

It is surprising to see that, for identical flow conditions, the higher regularity of the chains of iPP, i.e. higher crystallization ability, does not imply a higher total length per volume of oriented nuclei $L$ (Table 2). Notice that the long period $l$ of stacked lamellae (independent of shear) and nuclei density $N$ (dependent on shear) together determine the total length of oriented nuclei per volume $L=l \times N$. One could suggest that the difference in the long period for the three materials leads to the varying total length of oriented nuclei. However, Hosier et al. $^{56}$ used AFM and found that the long period at 110 
${ }^{\circ} \mathrm{C}$ decreases with addition of ethylene defects. On the basis of their data, the estimated long periods of our materials at $110{ }^{\circ} \mathrm{C}$ change from $\sim 20 \mathrm{~nm}$ for iPP to $\sim 15 \mathrm{~nm}$ and $\sim 10 \mathrm{~nm}$ for random copolymer with $3.4 \mathrm{~mol} \%$ and $7.3 \mathrm{~mol} \%$ ethylene monomer, respectively. Even though the long period at $137^{\circ} \mathrm{C}$ might be different from the above numbers for $110^{\circ} \mathrm{C}$, it may still be concluded that the lower total nuclei length for iPP is not caused by its larger long period, because the long period, $l$, decreases with increase of ethylene content and results in a larger difference in nucleation number, $N=L / l$.

The influence of the stress history on crystallization is determined by the response of a polymer at the molecular level, i.e. the molecular stretch. ${ }^{22}$ As described by the nucleation and growth model, ${ }^{22}$ the total length of nuclei is determined by both the rate of generation of new nuclei and the rate of longitudinal growth of these nuclei during shear. The nucleation rate depends on the stretch $\Lambda_{H M W}$ of the high molecular weight (HMW) tail and the longitudinal growth rate on the average molecular stretch $\Lambda_{A V G}$. Therefore, the high molecular weight tail and average stretch play different roles in increasing the amount of oriented nuclei. For a pressure-driven flow device as used in the present work, imposing the same shear stress ensures that the average stretch is the same, irrespective of ethylene content in polymer. However, a difference in stretch history of the HMW tail may cause a significant change in nuclei quantity. In fact, the reptation time of the HMW tail in the RACO's, determined from dynamic rheological measurements, is larger than for the iPP homopolymer; 1.46, 2.07, and 3.14 s for iPP, RACO3, and RACO7 at 220 ${ }^{\circ} \mathrm{C}$, respectively. ${ }^{35}$ Since the temperature dependence of the reptation time follows the Arrhenius equation, the longest relaxation times at $137^{\circ} \mathrm{C}$ can be calculated according to $\left(\tau_{T}\right) /$ $\left(\tau_{r e f}\right)=\exp \left[-E / R\left(1 / T-(1) /\left(T_{\text {ref }}\right)\right)\right]$ with activation energy $E$ (43.0, 42.04, and $45.19 \mathrm{~kJ} / \mathrm{mol}$ for iPP, RACO3, and RACO7, respectively) ${ }^{35}$ and universal gas constant $R$. It is found that the reptation times of HMW tails in RACO3 and RACO7 are 16.5 and $29.2 \mathrm{~s}$ at $137^{\circ} \mathrm{C}$, respectively, which are 1.4 and 2.4 times larger than that of $12.2 \mathrm{~s}$ in iPP. This means that compared to the homopolymer, the corresponding Rouse times of the HMW tail are also larger for the RACO's. Therefore, for the same imposed stress, the high molecular weight tail is able to attain somewhat higher degrees of orientation and stretch during flow for the materials with higher ethylene content. Previous studies have found enhanced flow-induced crystallization under conditions in which the longest polymer chains can stretch. ${ }^{17,} 18,58-60$ Therefore, for our materials, we expect a specific level of stress - i.e. at a given layerto cause a larger influence on crystallization with increasing ethylene content, which is consistent with our estimation of oriented nuclei densities shown in Table 2.

The series of polymers studied here have a different ethylene monomer content and a different high molecular weight (HMW) tail, the latter resulting in dissimilar reptation and Rouse times of the HMW tail. It is known that presence of random ethylene co-units lowers the crystallization capability. However, it is also known that the HMW tail can enhance the effect of flow on nucleation, so the question of which one dominates flow-induced crystallization arises. Flow-induced crystallization under mild conditions has already been studied on the same materials ${ }^{35}$ and demonstrates that at fixed nominal undercooling (temperature difference between the experimental temperature and nominal melting temperature), the presence of ethylene monomer dominates the formation of pointlike nuclei and the transition from point-like nuclei to oriented nuclei. It was also found that increased ethylene content de- creases the effect of a given shear rate on nucleation (see Figure 8 in Reference 35: Housmans, Peters, \& Meijer, J. Therm. Anal. Calorim. 98:3 (2009), pp. 693-705), although the HMW tails in random copolymers have longer relaxation times. Differently, the current work under conditions of high shear stress (in the order of $0.1 \mathrm{MPa}$ ) shows that the formation of flow-induced oriented nuclei is dominated by the HMW tail and not by the ethylene content, in spite of the fact that at fixed crystallization temperature, the degree of undercooling is smaller for the random copolymers than for the homopolymer. Therefore, the role of molecular regularity and high molecular weight tail in dominating nucleation depends on the flow strength.

Although the total length per volume of nuclei is higher for the random copolymers, the growth rate of random copolymers is much lower than that of iPP. For 2D growth, crystallization kinetics is determined by the total length of nuclei and the square of growth rate, $\Phi(t)=1-\exp \left(-\pi L G^{2} t^{2}\right)$, so the overall crystallization kinetics is still dominated by the growth rate and decreases with increasing ethylene content.

\section{Conclusions}

Using a pressure-driven slit flow device and the depth sectioning method, the crystallization of an iPP homopolymer and two random copolymers with 3.4 and $7.3 \mathrm{~mol} \%$ ethylene was studied. For a given material, the crystallization rate increases from the inner to outer layers because of the linearly increasing level of stress from the center of the channel up to the wall. Once crystallization starts, the emergence of daughter lamellae is observed later than the development of parent lamellae. The greater length of oriented nuclei per unit volume in the outermost layer leads to a higher degree of orientation due to reduced space between neighboring nuclei, i.e., due to the decreased space available for lamellar curving and twisting. The highest level of stress examined can generate up to $10^{11} \mathrm{~m} / \mathrm{m}^{3}$ of oriented nuclei length density and even allows the crystallization of RACO7 to be detected in the vicinity of its nominal melting temperature. Flow-induced nuclei are quantified using kinetic analysis, and the results show that the total length of nuclei per volume in the iPP homopolymer is lower than that in the random copolymers. The increase of nuclei length per volume with ethylene may be explained by the larger relaxation times of the high molecular weight tail found for the random copolymers and therefore, of the corresponding Rouse time, which determines the molecular stretch of the longest molecules and thus the nucleation rate (as given by the "nucleation and growth model"). However, since the growth rate is reduced significantly by adding ethylene monomer, the overall crystallization kinetics, dominated by growth, is still faster for the homopolymer than for the random copolymers, in spite of having less oriented nuclei length per volume.

\section{Supporting Information}

"Estimation of growth rates at $137^{\circ} \mathrm{C}$ for all materials" is presented following the References.

Acknowledgments - We thank Dr. Markus Gahleitner (Borealis, Austria) for providing the polymer materials. NWO (Nederlandse Organisatie voor Wetenschappelijk Onderzoek) and ESRF are acknowledged for granting the beamtime. L.F.B thanks Prof. H. E. H. Meijer and Prof. A. J. Ryan for funding. We are also indebted to Dr. Wim Bras for valuable experimental advice and discussions. This work is part of the research 
program of the Dutch Polymer Institute (DPI), P.O. Box 902, 5600 AX Eindhoven, The Netherlands, Project Number 714. The authors declare no competing financial interest.

\section{References}

1. Pennings, A.; Kiel, A. Colloid Polym. Sci. 1965, 205 (2) 160-162

2. Keller, A. Philos. Mag. 1957, 2 (21) 1171-1175

3. Hsiao, B. S.; Yang, L.; Somani, R. H.; Avila-Orta, C. A.; Zhu, L. Phys. Rev. Lett. 2005, 94 (11) 117802

4. Eder, G.; Janeschitz-Kriegl, H.Structure development during processing: crystallization. In Processing of Polymers; Meijer, H., ed.; Wiley-VCH: Weinheim, Germany, 1997; Vol. 18, pp 269-342.

5. Keller, A.; Kolnaar, H. W. H. Flow-induced orientation and structure formation. In Processing of Polymers, Meijer, H., ed.; Wiley-VCH: Weinheim, Germany, 1997; Vol. 18, pp 189-268.

6. Kumaraswamy, G. J. Macromol. Sci., Part C: Polym. Rev. 2005, 45 (4) 375-397

7. Somani, R. H.; Yang, L.; Zhu, L.; Hsiao, B. S. Polymer 2005, 46 (20) 8587-8623

8. Langouche, F. Macromolecules 2006, 39 (7) 2568-2573

9. An, H.; Zhao, B.; Ma, Z.; Shao, C.; Wang, X.; Fang, Y.; Li, L.; Li, Z. Macromolecules 2007, 40 (14) 4740-4743

10. Vleeshouwers, S.; Meijer, H. E. H. Rheol. Acta 1996, 35 (5) 391-399

11. Pogodina, N. V.; Winter, H. H. Macromolecules 1998, 31 (23) 8164-8172

12. Liedauer, S.; Eder, G.; Janeschitz-Kriegl, H.; Jerschow, P.; Geymayer, W.; Ingolic, E. Int. Polym. Process. 1993, 8, 236-244

13. Kumaraswamy, G.; Verma, R. K.; Kornfield, J. A. Rev. Sci. Instrum. 1999, 70 (4) 2097-2104

14. Housmans, J. W.; Steenbakkers, R. J. A.; Roozemond, P. C.; Peters, G. W. M.; Meijer, H. E. H. Macromolecules 2009, 42 (15) 5728-5740

15. Pogodina, N. V.; Siddiquee, S. K.; van Egmond, J. W.; Winter, H. H. Macromolecules 1999, 32 (4) 1167-1174

16. Baert, J.; Van Puyvelde, P. Macromol. Mater. Eng. 2008, 293 (4) 255-273

17. Fernandez-Ballester, L.; Thurman, D. W.; Zhou, W.; Kornfield, J. A. Macromolecules 2012, 45 (16) 6557-6570

18. Balzano, L.; Kukalyekar, N.; Rastogi, S.; Peters, G. W. M.; Chadwick, J. C. Phys. Rev. Lett. 2008, 100 (4) 048302

19. Balzano, L.; Rastogi, S.; Peters, G. Macromolecules 2011, 44 (8) 2926-2933

20. An, H.; Li, X.; Geng, Y.; Wang, Y.; Wang, X.; Li, L.; Li, Z.; Yang, C. J. Phys. Chem. B 2008, 112 (39) 12256-12262

21. Geng, Y.; Wang, G.; Cong, Y.; Bai, L.; Li, L.; Yang, C. Macromolecules 2009, 42 (13) 4751-4757

22. Custodio, F. J. M. F.; Steenbakkers, R. J. A.; Anderson, P. D.; Peters, G. W. M.; Meijer, H. E. H. Macromol. Theory Simul. 2009, 18 (9) 469-494

23. Mackley, M. R.; Hassell, D. G. J. Non-Newtonian Fluid Mech. 2011, 166 (9-10) 421-456

24. Housmans, J. W.; Balzano, L.; Santoro, D.; Peters, G. W. M.; Meijer, H. E. H. Int. Polym. Process. 2009, 24 (2) 185-197
25. Ma, Z.; Balzano, L.; Peters, G. W. M. Macromolecules 2012, 45 (10) 4216-4224

26. Kumaraswamy, G.; Issaian, A. M.; Kornfield, J. A. Macromolecules 1999, 32 (22) 7537-7547

27. Fernandez-Ballester, L.; Gough, T.; Meneau, F.; Bras, W.; Ania, F.; Francisco, J. C.; Kornfield, J. A. J. Synchrotron Radiat. 2008, 15, 185-190

28. Kumaraswamy, G.; Verma, R. K.; Kornfield, J. A.; Yeh, F. J.; Hsiao, B. S. Macromolecules 2004, 37 (24) 9005-9017

29. Balzano, L.; Cavallo, D.; van Erp, T. B.; Ma, Z.; Housmans, J. W.; Fernandez-Ballester, L.; Peters, G. W. M. IOP Conf. Ser.: Mater. Sci. Eng. 2010, 14, 01200

30. Fernandez-Ballester, L.; Thurman, D. W.; Kornfield, J. A. J. Rheol. 2009, 53 (5) 1229-1254

31. Macosko, C. W., Rheology, principles, measurements and application; Wiley-VCH: New York, 1994.

32. Gahleitner, M.; Jääskeläinen, P.; Ratajski, E.; Paulik, C.; Reussner, J.; Wolfschwenger, J.; Neißl, W. J. Appl. Polym. Sci. 2005, 95 (5) 1073-1081

33. Maspoch, M. L.; Gamez-Perez, J.; Gimenez, E.; Santana, O. O.; Gordillo, A. J. Appl. Polym. Sci. 2004, 93 (6) 2866-2878

34. Gamez-Perez, J.; Muñoz, P.; Santana, O. O.; Gordillo, A.; Maspoch, M. L. J. Appl. Polym. Sci. 2006, 101 (5) 2714-2724

35. Housmans, J. W.; Peters, G. W. M.; Meijer, H. E. H. J. Therm. Anal. Calorim. 2009, 98 (3) 693-705

36. Jeon, K.; Chiari, Y. L.; Alamo, R. G. Macromolecules 2007, 41 (1) 95-108

37. Hosier, I. L.; Alamo, R. G.; Esteso, P.; Isasi, J. R.; Mandelkern, L. Macromolecules 2003, 36 (15) 5623-5636

38. Mykhaylyk, O. O.; Chambon, P.; Graham, R. S.; Fairclough, J. P. A.; Olmsted, P. D.; Ryan, A. J. Macromolecules 2008, 41 (6) 1901-1904

39. Bras, W.; Dolbnya, I. P.; Detollenaere, D.; van Tol, R.; Malfois, M.; Greaves, G. N.; Ryan, A. J.; Heeley, E. J. Appl. Crystallogr. 2003, 36, 791-794

40. Dean, D. M.; Rebenfeld, L.; Register, R. A.; Hsiao, B. S. J. Mater. Sci. 1998, 33 (19) 4797-4812

41. Housmans, J. W.; Gahleitner, M.; Peters, G. W. M.; Meijer, H. E. H. Polymer 2009, 50 (10) 2304-2319

42. Lotz, B.; Wittmann, J. C. J. Polym. Sci., Part B: Polym. Phys. 1986, 24 (7) 1541-1558

43. Somani, R. H.; Hsiao, B. S.; Nogales, A.; Fruitwala, H.; Srinivas, S.; Tsou, A. H. Macromolecules 2001, 34 (17) 5902-5909

44. Chen, Y.-H.; Mao, Y.-M.; Li, Z.-M.; Hsiao, B. S. Macromolecules 2010, 43 (16) 6760-6771

45. van Erp, T. B.; Balzano, L.; Peters, G. W. M. ACS Macro Lett. 2012, 1 (5) 618-622

46. Kumaraswamy, G.; Verma, R. K.; Issaian, A. M.; Wang, P.; Kornfield, J. A.; Yeh, F.; Hsiao, B. S.; Olley, R. H. Polymer 2000, 41 (25) 8931-8940

47. Janeschitz-Kriegl, H.; Ratajski, E.; Stadlbauer, M. Rheol. Acta 2003, 42 (4) 355-364

48. Cavallo, D.; Azzurri, F.; Balzano, L.; Funari, S. S.; Alfonso, G. C. Macromolecules 2010, 43 (22) 9394-9400

49. Kolmogorov, A. N. Bull. Acad. Sci. USSR. Ser. Math 1937, 3, 355-360 
50. Avrami, M. J. Chem. Phys. 1939, 7 (12) 1103-1112

51. Avrami, M. J. Chem. Phys. 1940, 8 (2) 212-224

52. Evans, U. R. Trans. Faraday Soc. 1945, 41, 365-374

53. Space filling refers to impingement, after which the secondary crystallization may happen. Therefore, the area value right after the impingement shoulder is used to indicate the finish of space filling, instead of the last value of the graph.

54. Wunderlich, B., Macromolecular Physics; Academic Press: New York, 1976; Vol. 2.

55. Binsbergen, F. L.; de Lange, B. G. M. Polymer 1970, 11 (6) 309-332
56. Hosier, I. L.; Alamo, R. G.; Lin, J. S. Polymer 2004, 45 (10) 3441-3455

57. Ma, Z.; Steenbakkers, R. J. A.; Giboz, J.; Peters, G. W. M. Rheol. Acta 2011, 50 (11-12) 909-915

58. Seki, M.; Thurman, D. W.; Oberhauser, J. P.; Kornfield, J. A. Macromolecules 2002, 35 (7) 2583-2594

59. van Meerveld, J.; Peters, G. W. M.; Hutter, M. Rheol. Acta 2004, 44 (2) 119-134

60. Steenbakkers, R. J. A.; Peters, G. W. M. J. Rheol. 2011, 55 (2) 401-433 


\title{
Supporting information
}

\section{High-stress shear induced crystallization in isotactic polypropylene and propylene/ethylene random copolymers}

\author{
Zhe Ma, Lucia Fernandez-Ballester, Dario Cavallo, Tim Gough, Gerrit W. M. Peters
}

1. The linear function $\left(\log (G)=a+b^{*} T\right)$ was used to fit experimental data of growth rates $(G)$ at different temperatures $(\mathrm{T})$. The parameters, $\mathrm{a}$ and $\mathrm{b}$, are given in Table S1.

Table S1. Fitting parameters and the estimated growth rates at $137^{\circ} \mathrm{C}$

\begin{tabular}{|c|c|c|c|}
\hline & $\mathrm{a}$ & $\mathrm{b}$ & growth rate at $137^{\circ} \mathrm{C}(\mathrm{nm} / \mathrm{s})$ \\
\hline iPP & 12.17 & 0.079 & 24.5 \\
\hline RACO3 & 12.24 & 0.085 & 4.4 \\
\hline RACO7 & 11.15 & 0.079 & 2.1 \\
\hline
\end{tabular}

The growth rates at $137{ }^{\circ} \mathrm{C}$ of iPP and RACO3 are interpolated with above function and parameters.

Since $137^{\circ} \mathrm{C}$ is too high to experimentally measure the quiescent growth rates, growth rate at $137^{\circ} \mathrm{C}$ of RACO7 was estimated by extrapolating the measured results. 